\title{
Analysis of Hospitalization Length of Stay and Total Charges for Patients with Drug Abuse Comorbidity
}

\author{
Memory Ndanga ${ }^{1}$, Shankar Srinivasan ${ }^{2}$ \\ 1. Health Information Management, Rutgers University, Piscataway, USA 2. Informatics, Rutgers University, \\ Piscataway, USA
}

Corresponding author: Memory Ndanga, ndangam@gmail.com

\section{Abstract \\ Background}

Drug abuse has been on the increase over the last few years, contributing to the healthcare cost. An understanding of the overall impact of drug abuse hospitalizations is essential in combatting the drug abuse epidemic.

\section{Objective}

To evaluate inpatient outcomes of total charges and length of stay for patients with drug abuse comorbidity compared to non-drug abuse admissions.

\section{Method}

The Healthcare Cost and Utilization Project (HCUP) Nationwide Inpatient Sample data was utilized. Drug abuse comorbidity was used as defined by HCUP. Various descriptive and inferential analyses were performed on the filtered data sets for the years 2010 to 2014.

\section{Results}

The average hospitalization length of stay was 4.5 days for non-drug abuse and 5.5 days for drug abuse comorbidity $(\mathrm{P}<0.001)$. Mean charges for drug abuse comorbidity were significant for claims to private insurance and Medicaid.

\section{Conclusion}

Total charges and length of stay are higher for drug abuse than non-drug abuse cases. The results will aid as a reference for resource allocation and policy changes. Further research is needed for alternative and

Received 11/07/2019

Categories: Miscellaneous, Public Health, Other

Keywords: drug abuse, comorbidity, length of stay, national inpatient sample, cost, inpatient days, drug misuse, drug dependence, hospitalization, total charges

\section{Introduction}

Drug abuse has increased in the past two decades, causing a substantial burden not only to the families but also to the healthcare system and the United States (US) economy as a whole. This study analyzes the outcome of the length of stay and total charges of patients with drug abuse comorbidity. Studies on drug abuse comorbidity are of importance in resource allocation and can be used as a necessary reference tool primarily as the nation is geared towards combatting the current drug abuse problem. The majority of people know someone with a substance use disorder or someone who has lost a relative or friend due to substance misuse. Drug abuse has become more prevalent, such that it is not a surprise that there were 27 million adult Americans who have self-reported the misuse of illegal drugs or opioid-based prescription drugs in 2015 [1]. The drug abuse hospitalization cost was over $\$ 21$ billion in 2010 and is expected to grow as the epidemic continues. The business sector has also identified the second largest expense for large and small businesses, apart from salary, being health care for their employees. Productivity losses related to personal and family health problems is estimated to cost US employers $\$ 1,685$ per employee per year, or $\$ 225.8$ billion annually [2], of which drug abuse is a contributing factor. The total healthcare expenditure for 2014 was set to have increased by $4.1 \%$ [3]. Hospital admissions of drug-related diagnoses are potentially avoidable, saving the healthcare system a substantial amount of money and resources [4-5]. The Agency for Healthcare Research and Quality (AHRQ) reported that the national rate of drug abuse-related inpatient stays and emergency department (ED) visits increased 64.1\% and 99.4\%, respectively, in 2005 - 2014 [6]. Therefore, there is a need to analyze the drug abuse comorbidity pattern. 
In a cost comparison study that was initiated to calculate the excess burden of drug abuse, the drug abuse group had higher utilization rates for medical services and prescription drugs. The study identified patients with drug abuse using medical and pharmacy claims data. The results were compared with non-opioid abuse diagnosis using linear regression. Drug abusers had an eight times higher cost than non-abusers. Hospital inpatient visits for opioid abusers were 12 times higher than non-abusers, resulting in an estimated mean annual cost of at least $\$ 15,884$ compared to $\$ 1,830$, respectively [7]. At least one in every five days was attributed to substance abuse stay, and 1.2 million days were used for direct treatment of substance abuse, i.e., treating diseases wholly attributable to substance abuse. With the constant increase in medical costs, policies are focusing on reducing resource consumption by decreasing the number of inpatient days [8-9]. Marijuana studies also examined the incremental inpatient costs of treatment that showed that marijuana comorbidity was associated with longer length of stays and higher charges [10].

\section{Materials And Methods}

For this project, data was taken from the National Inpatient Sample (NIS), which is part of the Healthcare Cost and Utilization Project (HCUP). HCUP is associated with the AHRQ. HCUP combines both private and state organization data, including the Federal government. It is the most extensive collection of longitudinal hospital data. All-payer and encounter levels information is also provided. Researchers have been able to utilize this database for research on a broad range of healthcare policies, including qualities on the cost of services, access to healthcare and programs, and outcomes of treatment at all levels.

This study includes comorbidity drug abuse cases from the 2010 - 2014 HCUP National Inpatient Sample Database [11]. The design for this quantitative approach included a thorough analysis of hospitalization outcomes for the selected years. The IBM Statistical Package for Social Sciences (SPSS) (IBM SPSS Statistics, Armonk, NY) and Microsoft ${ }^{\circledR}$ Excel (Microsoft ${ }^{\circledR}$ Corp., Redmond, WA) were used in the analysis and preparation of the data. Drug abuse comorbidity cases were filtered for data analysis. Various descriptive and inferential analysis were performed on the filtered data sets for the above years.

\section{Results}

The study sample included a total of 2,258,235 drug abuse comorbidity patients, of which $54 \%$ were males and $46 \%$ were females $(\mathrm{p}<0.001)$. The age range for patients with drug abuse comorbidity varied, as shown in Figure 1 below. In order to better understand the differences in age groups, the patient ages were divided into five groups ranging from 0 - 81 and older as shown in Figure 1 . The mean age of the study population was 44.45 with a standard deviation (SD) of 6.72 years (mean age for females was 43 years, while males were 45 years, $\mathrm{p}<0.05)$

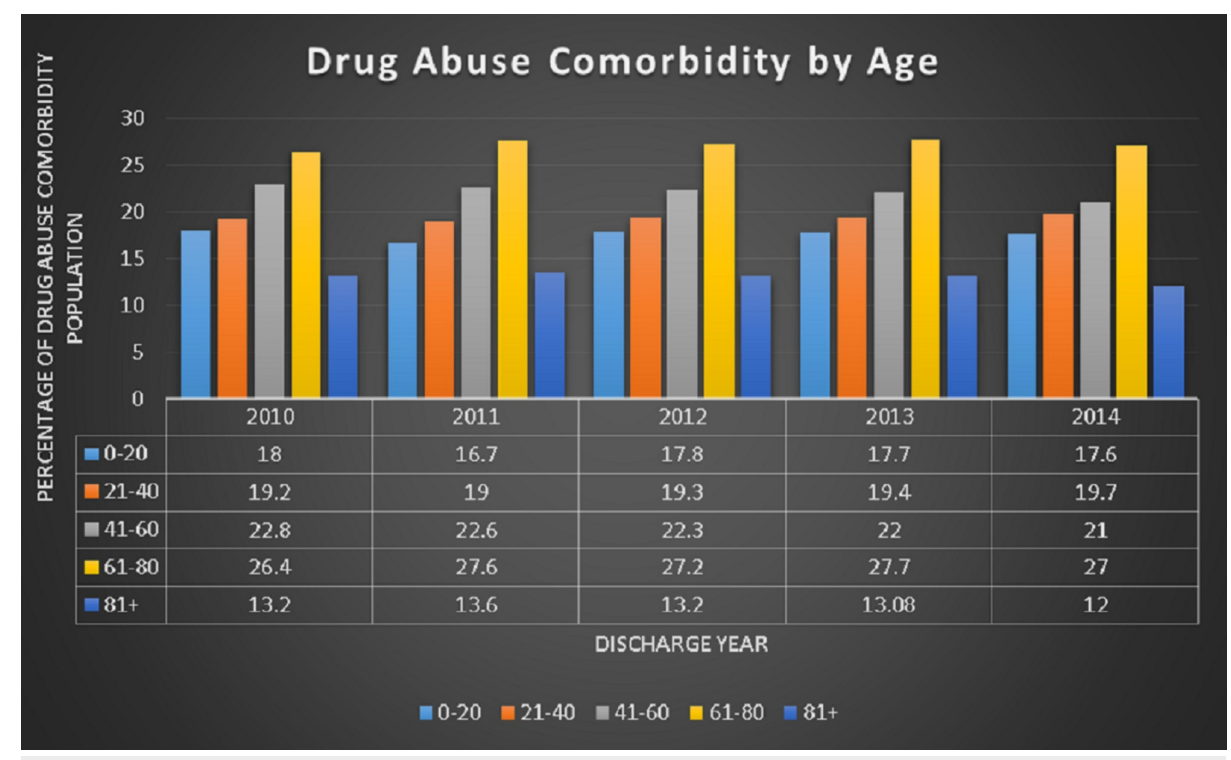

FIGURE 1: Age groups of drug abuse cases

CM: comorbidity

Figure 2 shows the gender difference for patients with drug abuse comorbidity; $54 \%$ were males and $46 \%$ were females $(\mathrm{p}<0.001)$. 


\section{Cureus}

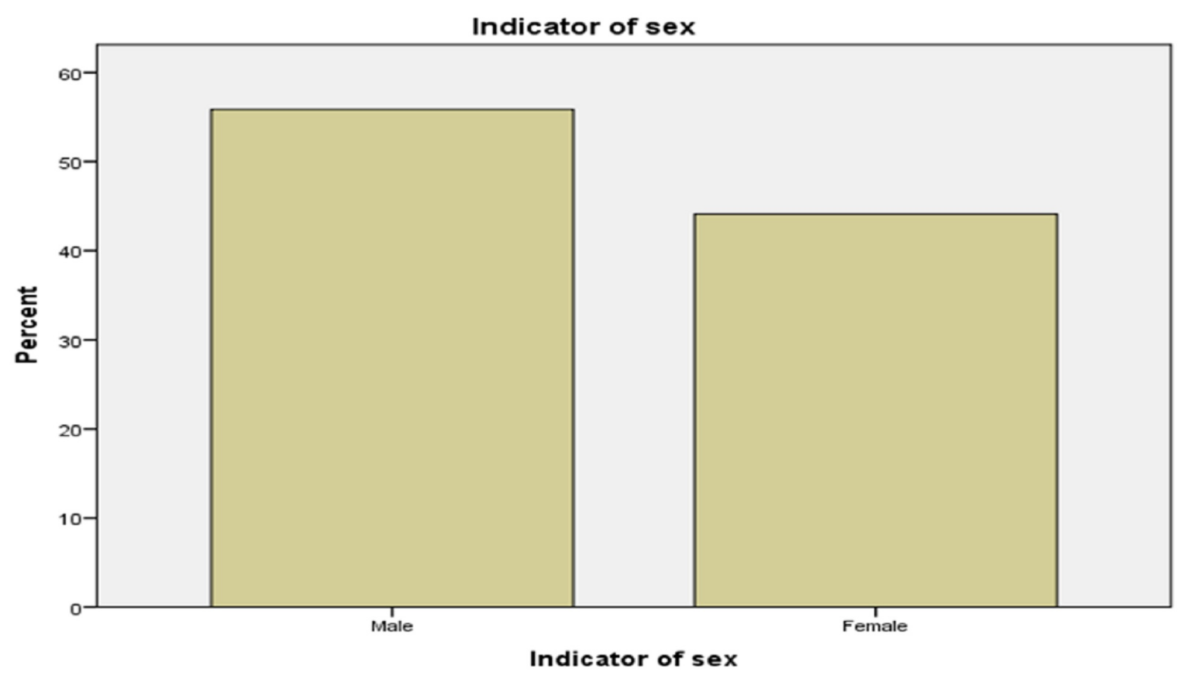

FIGURE 2: Gender and Drug Abuse Comorbidity

A total of 629,608 drug abuse comorbidity patients were White, which comprised $54.9 \%$ of the total, followed by Black (24.3\%), Hispanic (8.9\%), Asian (0.8\%), Native American (0.8\%), and others $(2.5 \%)$. The race distribution is shown in Figure 3.

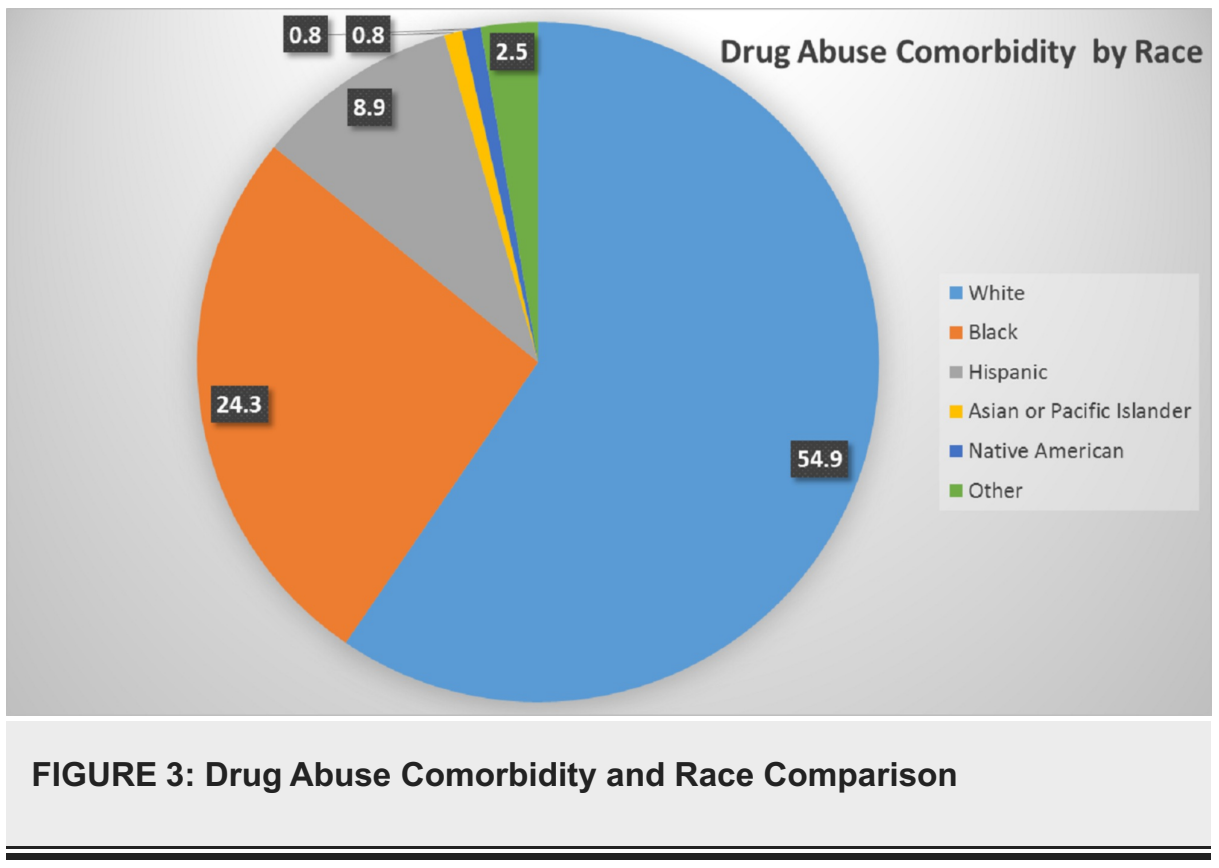

Table 1 shows the primary expected payer and that Medicaid was the main form of health insurance charged $36 \%$. Medicare had $22.6 \%$ while private and self-pay had 18.2 and 15.1, respectively and other not specified insurance types were charged $5.4 \%$. 


\section{Cureus}

\begin{tabular}{|c|c|c|c|}
\hline & & Frequency & Percent \\
\hline \multirow{6}{*}{ Valid } & Medicare & 510,179 & 22.6 \\
\hline & Medicaid & 827,936 & 36.7 \\
\hline & Private & 411,272 & 18.2 \\
\hline & Self-pay & 341,257 & 15.1 \\
\hline & No Charge & 37,369 & 1.7 \\
\hline & Other & 122,357 & 5.4 \\
\hline Total & & $2,258,235$ & 100 \\
\hline
\end{tabular}

\section{TABLE 1: Shows Primary Expected Payer}

When comparing the length of stay for between drug abuse comorbidity patient and non-drug abuse, the average stay for a patient with drug abuse comorbidity was 5.52 compared to 4.56 for non-abuse as shown in Table 2 .

\begin{tabular}{|l|c|c|}
\hline Drug abuse & Mean & Number \\
\hline Comorbidity Drug Abuse Not Present & 4.56 & $286,979,236$ \\
\hline Comorbidity Drug Abuse Present & 5.52 & $11,211,054$ \\
\hline Total & 4.59 & $298,190,290$ \\
\hline
\end{tabular}

\section{TABLE 2: Length of Stay Comparison for Comorbidity Drug Abuse and Non-drug Abuse}

SD: standard deviation

The length of stay comparison by gender is shown in Figure 4 reflects that males with drug abuse comorbidity had an average of 5.7 inpatient days, while male non-drug abuse patients stayed for 4.9 days. Females who had drug abuse comorbidity had an average of 5.2 days, while female non-drug abuse patients had 4.3 inpatient days. The $\mathrm{p}$-value was significant at $\mathrm{p}<0.001$

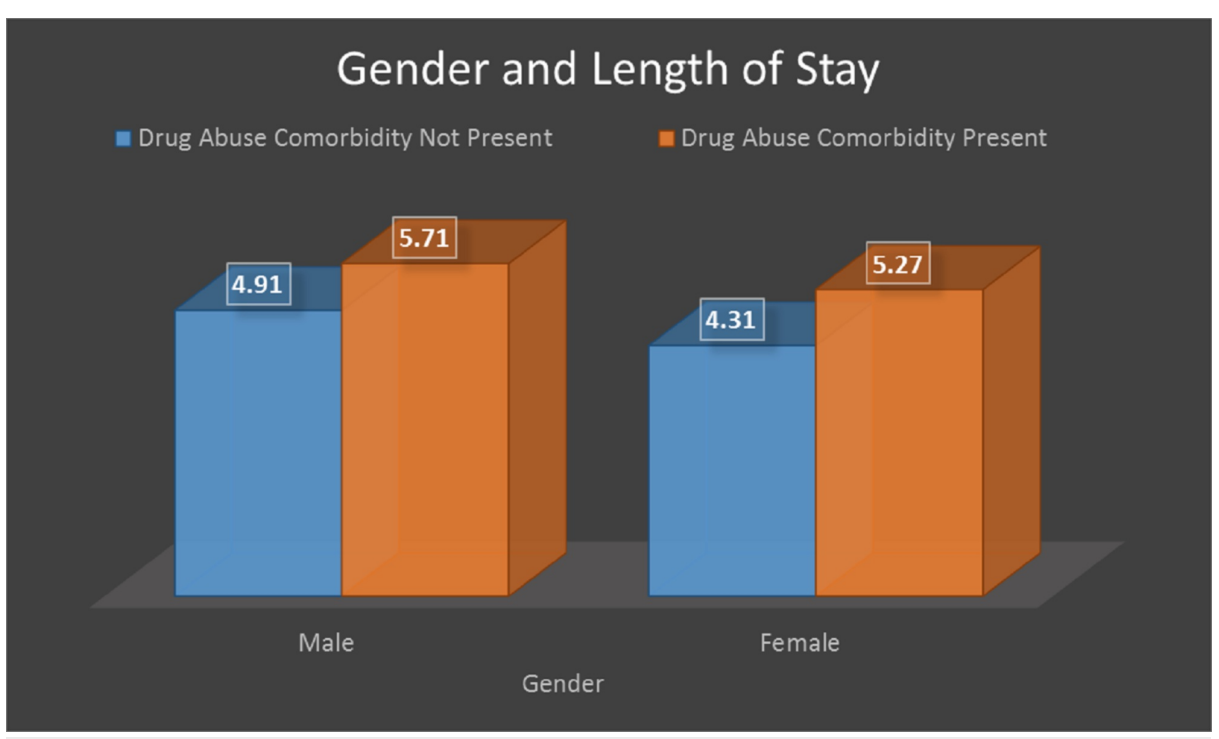

FIGURE 4: Length of stay comparison by gender 


\section{Cureus}

The length of stay comparison between races shown in Figure 5 varied. The range of the inpatients days were 4.1 - 4.6 days for non-drug abuse cases, while drug abuse comorbidity ranged from 5.2 - 6.5 days, as shown in Figure 5. The $\mathrm{p}$-value for race and length of stay was significant at $\mathrm{p}<0.001$ and a $95 \%$ confidence interval.

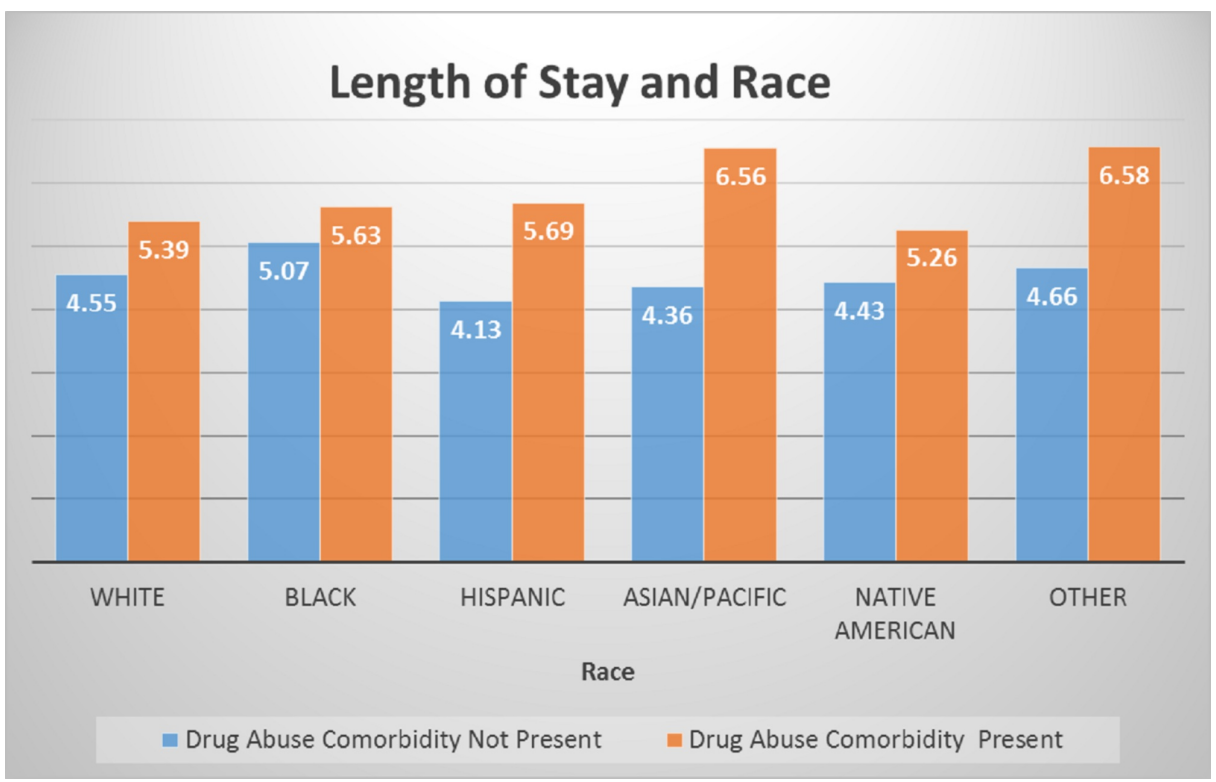

FIGURE 5: Length of stay comparison by race

Classification software diagnosis categories were used to identify the relationship between the length of stay and diagnosis categories. Figure 6 shows that the lowest inpatient days were recorded for obstetrics nondrug abuse of 2.6 days and 3.1 for drug abuse comorbidity. The most extended stay for non-drug abuse was 7.6 days for the infectious category, while the highest for drug abuse was for a perinatal category that had a total inpatient stay of 43.2 days.

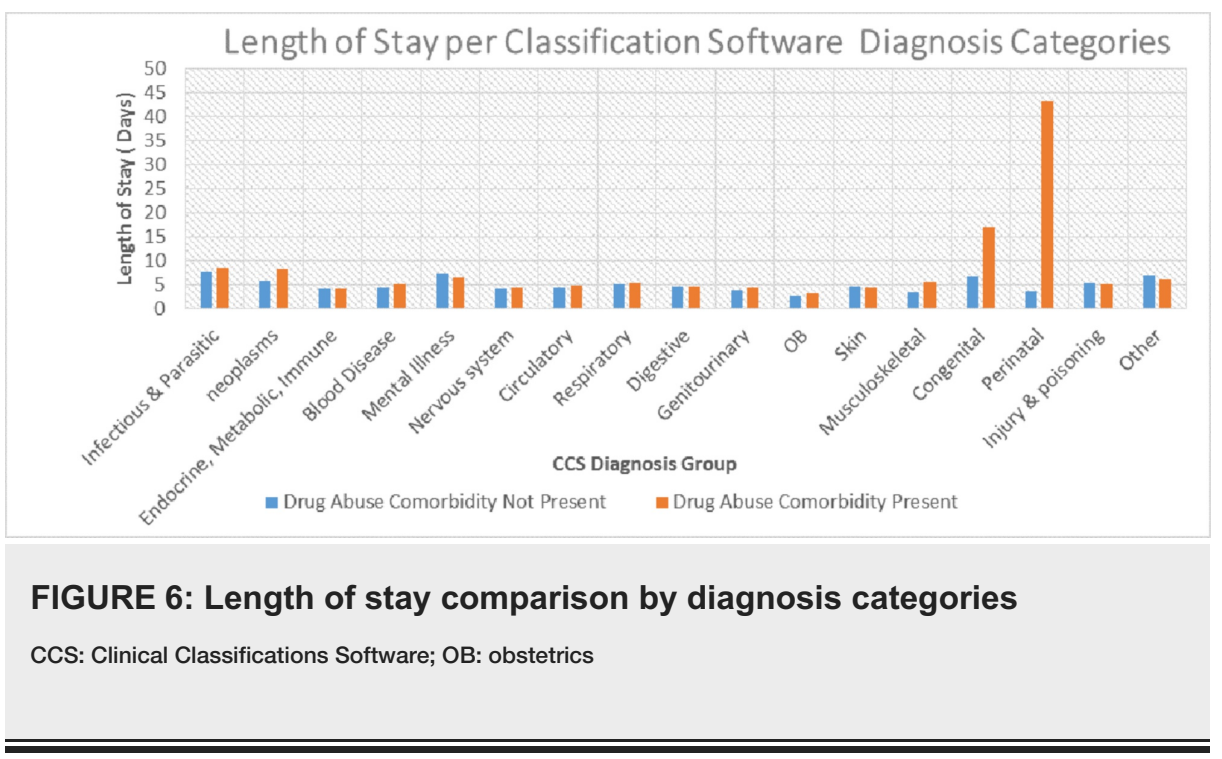

Figure 7 shows the association of the length of stay and type of insurance. Medicare patients with drug abuse comorbidity spent six days compared to five days for non-drug abuse. The same trend was seen for all insurance types that insurance companies have to pay for a day more for drug abuse comorbidity patients compared to non-drug abuse. 


\section{Cureus}

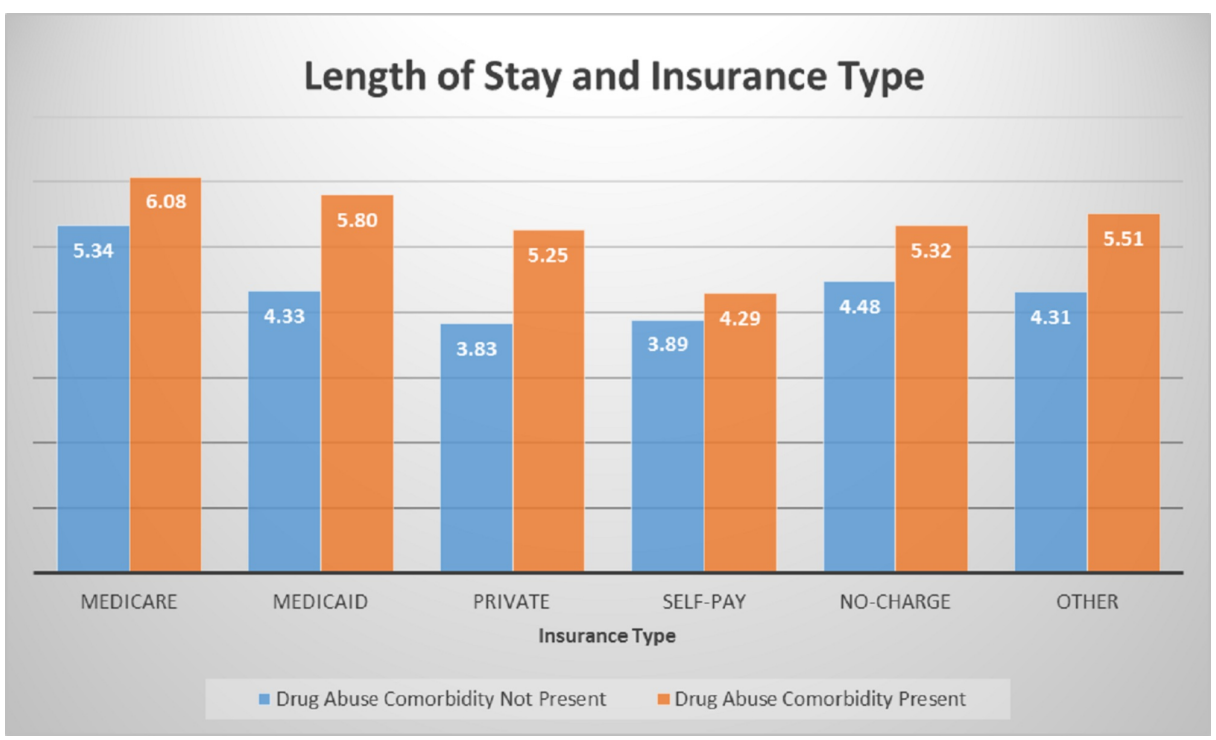

\section{FIGURE 7: Length of stay comparison by insurance type}

Significant $p$-values $<0.05$ at $95 \%$ confidence interval

The mean charge for each age group shown in Figure 8. The 0 - 20-year-old age group of non-drug abuse cases had $\$ 20,000$ in charges, and the drug abuse comorbidity cases had $\$ 34,000$. The 21 - 40-year-old age group had $\$ 25,000$ for non-drug abuse, while the drug abuse comorbidity had $\$ 28,000$. The 41 - 60 -year-old age group had $\$ 44,000$ for non-drug abuse, while the drug abuse group had $\$ 38,000$. Older adults aged 61 80 years had $\$ 48,000$ for drug abuse comorbidity and the non-drug abuse group had $\$ 47,000$. Lastly, 81 years old and above had $\$ 42,000$ drug abuse comorbidity and $\$ 38,000$ for non-drug abuse cases. The results were significant at $\mathrm{p}<0.001$ and $95 \%$ confidence interval.

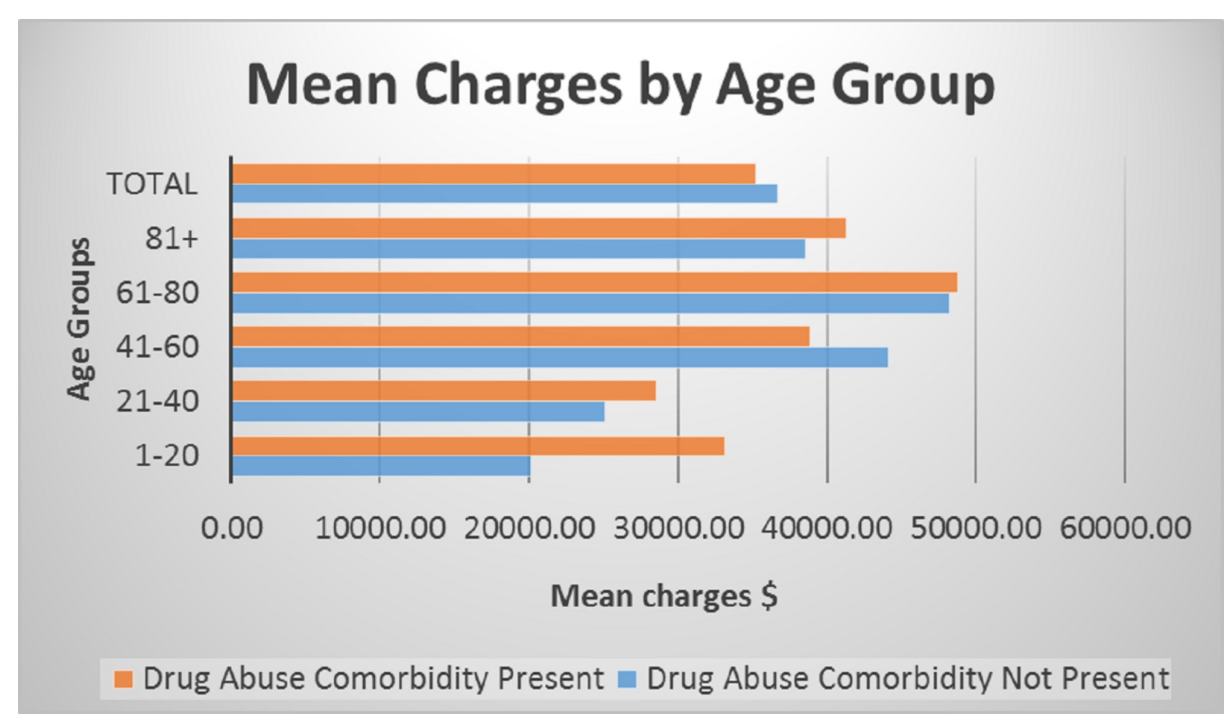

\section{FIGURE 8: Hospital charges comparison by age group}

The mean charges for race in Figure 9 show that for non-drug abuse, Caucasians had $\$ 38,000$ and $\$ 35,000$ for the drug abuse group. Blacks had $\$ 36,000$ for non-drug abuse group, while the drug abuse comorbidity group had $\$ 34,000$. Hispanics showed $\$ 44,500$ for the drug abuse comorbidity group and less than $\$ 40,000$ for nondrug abuse group. The Asian/Pacific Islander race showed higher charges at $\$ 47,000$ for the drug abuse comorbidity group and $\$ 41,000$ for the non-drug abuse group. Native Americans and Others, not specified race had $\$ 34,000$ and $\$ 39,000$, respectively, for the drug abuse comorbidity group and the non-drug abuse group had $\$ 33,000$ and $\$ 36,000$, respectively. There was a significant P-value of less than 0.001 . 


\section{Cureus}

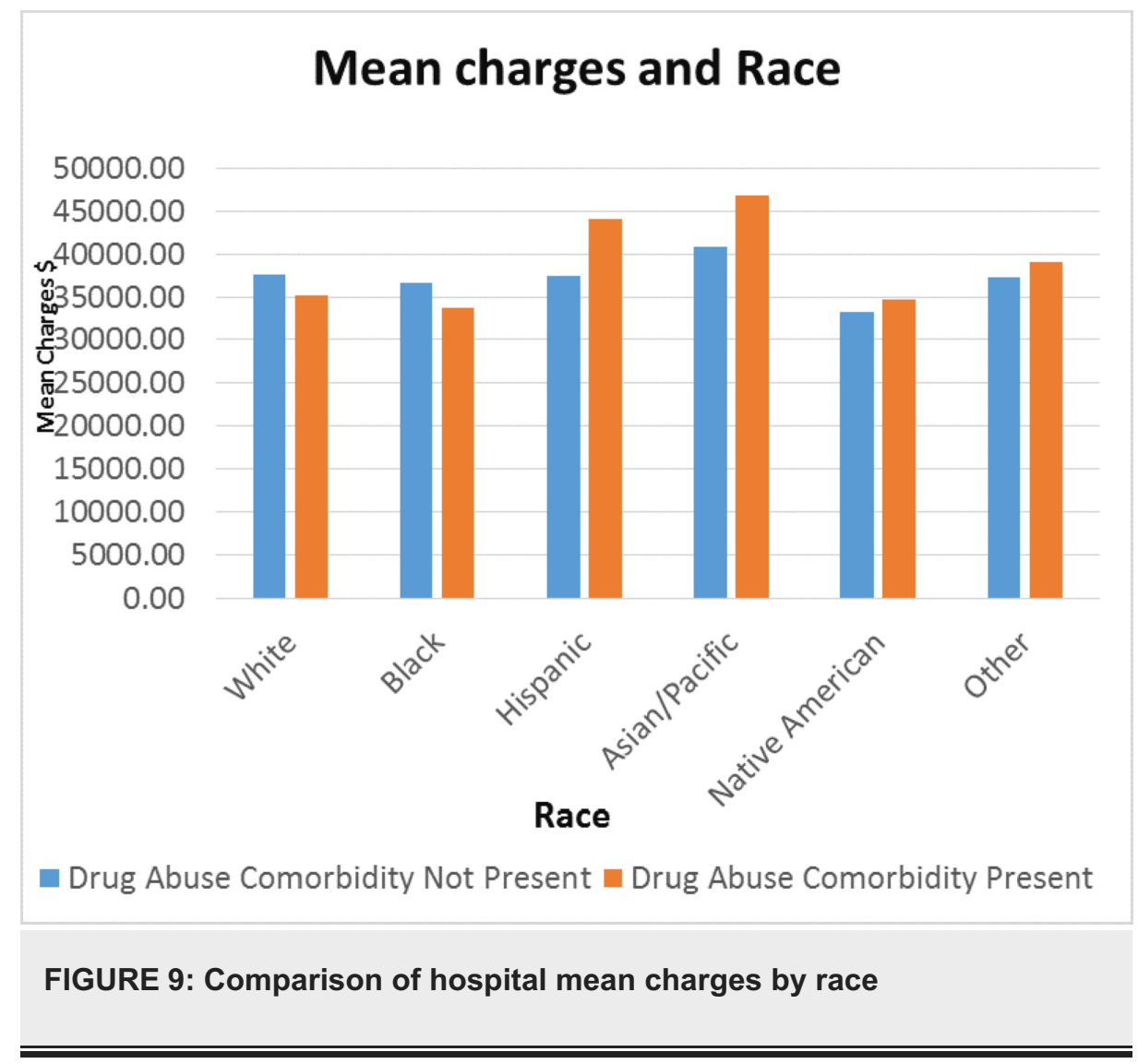

Figure 10 shows Medicaid and private insurance paid more for drug abuse comorbidity than non-drug abuse cases. Medicaid recorded charges for patients with drug abuse comorbidity at $\$ 35,000$ compared to non-drug abuse charges of $\$ 26,500$ for the same insurance type. Private insurance also showed significant charges for drug abuse comorbidity cases at $\$ 36,000$ compared to non-drug abuse cases at $\$ 33,450$.

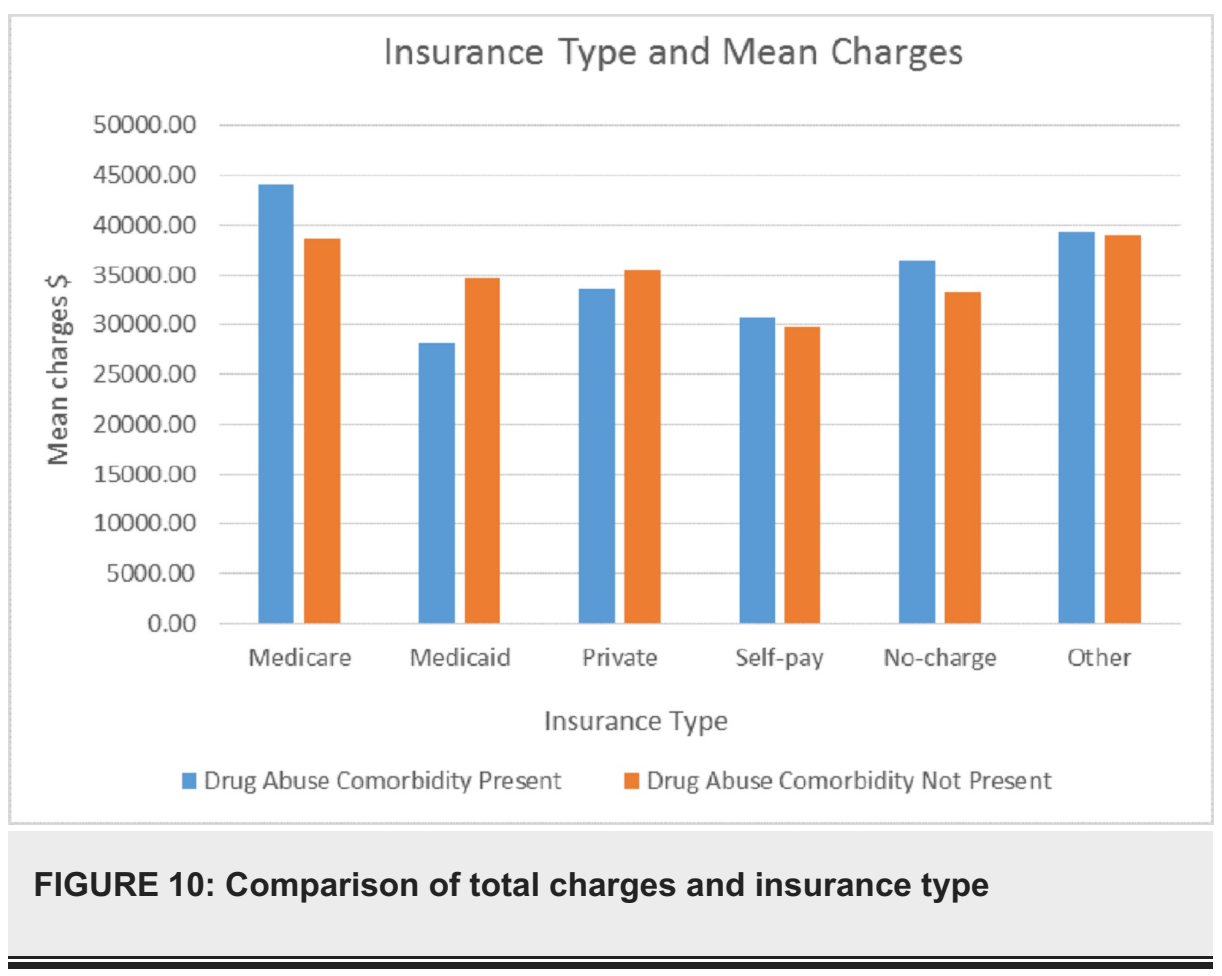

\section{Discussion}

In this retrospective study, we demonstrated that most of the drug abuse comorbidity patients were Caucasian males who were 44 years of age. These findings support the findings of other publications that 
have indicated that men are more likely than women to use almost all types of illicit drugs [12]. Men, in general, have a higher rate of drug and alcohol use compared to women [13]. We found substantial differences between races; Caucasians recorded the highest number of patients admitted with drug abuse comorbidity at $54 \%$, while Blacks and Hispanics had $24 \%$ and $9 \%$, respectively. All other races had less than $10 \%$ of the patients that were admitted with drug abuse comorbidity. In other studies that have looked at the different types of drugs in trauma settings, Caucasian and Hispanic patients were more likely to be admitted [14-15]. Blacks in rural areas were less likely to use methamphetamine compared to whites [16-17]. This is probably because each race is likely susceptible to a different type of drug, hence the need to expand the research on the types of drugs affecting each race. The US population surveys and treatment studies also indicated that there is a racial and ethnic difference in the prevalence of substance use [18]. There is also evidence that racial differences also result in minorities less likely to receive a variety of medical services. These disparities are significant even when looking at insurance status, income, age, and education level [19] and are more intensified when drug abuse and other mental disorders are present [20].

The most significant percentages of drug abuse-related stays were for patients within the two age groups, 41 - 60 and 61 - 80, which had an average of $22 \%$ and $27 \%$, respectively. Although older adults might not typically be considered drug seekers, it is likely that as their primary or secondary conditions become more clinically complicated, the continuous use of prescription medication might lead to addiction misuse or dependence. Older adults are also fragile and are susceptible to multiple factors. Other studies have also considered the potential of drug interactions associated with multiple conditions [21-22].

Maternal drug use also contributed to cases of babies that were less than two years to be included in the drug abuse population. Drug abuse comorbidity for infants is more costly and complicated. Substantial progress for the baby that has been exposed to drugs is very complicated and is attributed to the longer length of stay $[11,23]$.

It is also necessary that healthcare providers should consider the complete medical and social factors contributing to healthcare as abuse intensifies existing conditions [24-25]. A higher proportion of patients with drug abuse comorbidity showed that the most prevalent principal diagnosis was depression and mood disorders. Other forms of psychosis were also noted to be very common in patients with drug abuse comorbidity. This correlates with other previous studies that have identified a similar pattern of cooccurrence of substance-related disorders and personality or mood disorders [26-27].

The need for prophylactic vaccination was also another common diagnosis for a patient with drug abuse comorbidity compared to non-drug abuse. This is possible to prevent infections related to injection drug use. Injection site studies have identified the increase in drug use-related infections and attributed them to a significant mortality and morbidity rate and a substantial hospitalization cost [28].

The mean length of stay was five days for both males and females. The longer the substance abuse patients were admitted, the more resources they were likely to use. An article by Heslin et al. found an association between specific drug abuse diagnoses with longer lengths of stay [29]. Table 2 shows that the mean hospital length of stay was a day longer in drug abuse comorbidity patients compared to non-drug abuse patients (4.56 versus 5.52 days with a p-value $<0.00$ ). An additional day of inpatient care increases resource utilization and adds to the overall cost of hospitalization. A more comprehensive and intervention targeted for drug abuse-related diagnosis could potentially reduce the length of stay. The Clinical Classifications Software (CCS) diagnosis category analysis showed that perinatal and congenital categories have an extremely higher length of stay when drug abuse comorbidity is present (43.2 days and 16.8 days, respectively) compared to 6.7 and 3.7 days, respectively, when there is no drug use. Respiratory and hemic/lymphatic procedures also showed a longer length of stay when drug abuse comorbidity was present (12 and 11 days, respectively) compared to general non-abuse (nine days).

The study demonstrates the contribution of drug abuse comorbidity to the overall health service utilization. There was a significant difference in total charges for cases that reflected drug abuse comorbidity compared to non-drug abuse. When identifying charges for each age group, there were variations in total charges for each variable of interest. Total charges for the group aged 1 - 20 years were high for drug abuse comorbidity cases $(\$ 33,000)$ compared to the non-drug abuse group $(\$ 20,000)$. The young adults aged $21-40$ showed higher charges for drug abuse comorbidity cases with $\$ 28,100$ compared to $\$ 24,600$ for non-drug abuse. The older adults had mean charges of $\$ 38,000$ for non-drug abuse patients compared to $\$ 42,000$ for the drug abuse comorbidity patients.

The mean total charges, according to race, also showed that Hispanics and Asian/Pacific Islanders had higher hospital charges when they had a drug abuse comorbidity on record, even though they had fewer cases of drug abuse comorbidity admissions. This could be due to cultural differences and hesitation in seeking immediate treatment for their condition. However, when they do seek treatment, the treatment charges are likely to be higher because of being admitted through the emergency department or for treatment for intoxication and inpatient stays, resulting in additional hospitalization costs. Lack of insurance coverage has been linked to limited access to healthcare resources for minorities which is of significant concern as Hispanics, Blacks, and some Asian/Pacific Islander populations appear to have lower levels of 
The primary health insurance for the sample admissions was mostly either Medicaid, private insurance, or those without health insurance which probably could also be that the health insurance was not listed during the time of discharge. Medicaid covered more than $36 \%$ of the total patients admitted with drug abuse, followed by Medicare 23\%. This reflects that there is a significant burden on government-related health insurance benefits. This also possibly indicates the socioeconomic background of drug abuse patients. Medicare charges are the highest for drug abuse cases compared to general hospitalizations, which is in correlation with other Medicare and Medicaid cost studies [30].

To effectively provide information on charges and length of stay for drug abuse comorbidity, the Clinical Classification Software (CCS) for the International Classification of Diseases, Ninth Revision, Clinical Modification (ICD-9-CM) diagnosis and procedure categorization scheme was used. CCS categories have over 14,000 diagnosis codes and 3,900 procedure codes, which are collapsed into a smaller number of clinically meaningful categories that are more useful for presenting descriptive statistics than are individual ICD-9-CM codes. The CCS for ICD-9-CM, a diagnosis and procedure categorization analysis of charges, shows that certain conditions, such as perinatal and congenital diagnoses, will increase charges significantly when associated with drug abuse comorbidity compared to non-drug abuse cases. All CCS procedure categories also reflected an increase in charges when drug abuse comorbidity was present, suggesting the need for innovative interventions that are focused on addressing diagnosis and procedures that are coexisting with drug abuse comorbidity. Drug abuse comorbidity contributes to higher charges; therefore, linking the directly related diagnosis can provide a care plan that will reduce resource utilization and cost and improve patient clinical outcomes.

Our results depend on accurate coding of the diagnosis using the ICD-9-CM. The administrative database is an excellent resource for healthcare research, but as with any data, it is subject to coding inaccuracies and deficiencies. The lack of detailed information on patient progress during inpatient care limits the understanding of the length of stay causes, including readmission rates. The lack of cost breakdown on proportionality on total charges or costs limits the ability to stratify costs further. Despite these limitations, the data source is a large and nationally representative database that allows information to be captured from a majority of admissions and discharges in the US. The data includes all regions and covers a significant number of factors that allow a generalizable analysis of all hospitalizations in the US.

\section{Conclusions}

This study brings an understanding of the hospitalization outcomes of drug abuse comorbidity patients. We identified that charges are higher for drug abuse comorbidity than general admissions, drug abuse comorbidity hospitalization cases have a longer length of stay than non-drug abuse cases, and there are variations in age, race, and insurance payer for drug abuse comorbidity and non-drug abuse hospitalizations. Patients with drug abuse comorbidity have been noted to have other conditions that have resulted in a costlier inpatient stay, suggesting a more targeted approach for patient care for those who fall under the CCS diagnosis category of perinatal, congenital, and mood and personality disorders. Hospital mean charges for government-related insurances is also of the importance to policymakers who can evaluate potential costs and estimate budgets. Continuous research is needed to understand substance abuse comorbidity hospitalization in a more comprehensive approach, including identifying alternative and innovative interventions for conditions that are identified to be coexisting with drug abuse comorbidity.

\section{Additional Information \\ Disclosures}

Human subjects: Consent was obtained by all participants in this study. N/A issued approval N/A. HCUP Databases are Limited Data Sets. HCUP databases conform to the definition of a limited data set. A limited data set is healthcare data in which 16 direct identifiers, specified in the Privacy Rule, have been removed. Under HIPAA, review by an Institutional Review Board (IRB) is not required for use of limited data sets. Animal subjects: All authors have confirmed that this study did not involve animal subjects or tissue. Conflicts of interest: In compliance with the ICMJE uniform disclosure form, all authors declare the following: Payment/services info: All authors have declared that no financial support was received from any organization for the submitted work. Financial relationships: All authors have declared that they have no financial relationships at present or within the previous three years with any organizations that might have an interest in the submitted work. Other relationships: All authors have declared that there are no other relationships or activities that could appear to have influenced the submitted work.

\section{References}

1. Results from the 2016 National Survey on Drug Use and Health: Detailed Tables Prevalence Estimates, Standard Errors, P Values, and Sample Sizes. (2017). Accessed: December 20, 2019: http://www.samhsa.gov/data/sites/default/files/NSDUH-DetTabs-2016/NSDUH-DetTabs-2016.pdf.

2. Stewart WF, Ricci JA, Chee E, Morganstein D: Lost productive work time costs from health conditions in the United States: results from the American Productivity Audit. J Occup Environ Med. 2003, 45:1234-46. 
10.1097/01.jom.0000099999.27348.78

3. Martin AB, Hartman M, Benson J, Catlin A; National Health Expenditure Accounts Team: National health spending in 2014: faster growth driven by coverage expansion and prescription drug spending. Health Aff (Millwood). 2016, 35:150-60. 10.1377/hlthaff.2015.1194

4. McGeary KA, French MT: Illicit drug use and emergency room utilization. Health Serv Res. 2000, 35:153-69.

5. Masson CL, Sorensen JL, Batki SL, Okin R, Delucchi KL, Perlman DC: Medical service use and financial charges among opioid users at a public hospital. Drug Alcohol Depend. 2002, 66:45-50. 10.1016/s03768716(01)00182-x

6. Comorbidity: Addiction and Other Mental Diseases. (2010). Accessed: November 25, 2019: http://www.drugabuse.gov/sites/default/files/rrcomorbidity.pdf.

7. Birnbaum HG, White AG, Schiller M, Waldman T, Cleveland JM, Roland CL: Societal costs of prescription opioid abuse, dependence, and misuse in the United States. Pain Med. 2011, 12:657-67. 10.1111/j.15264637.2011.01075.x

8. Fulop G, Strain JJ, Vita J, Lyons JS, Hammer JS: Impact of psychiatric comorbidity on length of hospital stay for medical/surgical patients: a preliminary report. Am J Psychiatry. 1987, 144:878-82. 10.1176/ajp.144.7.878

9. Gryczynski J, Schwartz RP, O'Grady KE, Restivo L, Mitchell SG, Jaffe JH: Understanding patterns of high-cost health care use across different substance user groups. Health Aff (Millwood). 2016, 35:12-19. 10.1377/hlthaff.2015.0618

10. Pacula RL, Ringel J, Dobkin C, Truong K: The incremental inpatient costs associated with marijuana comorbidity. Drug Alcohol Depend. 2008, 92:248-57. 10.1016/j.drugalcdep.2007.08.011

11. Weiss AJ, Heslin KC, Barrett ML, Izar R, Bierman AS: Opioid-related inpatient stays and emergency department visits among patients aged 65 years and older, 2010 and 2015: Statistical Brief \#244 (Internet). Healthcare Cost and Utilization Project (HCUP) Statistical Briefs. Agency for Healthcare Research and Quality (US), Rockville; 2018. 50-56.

12. Results from the 2016 National Survey on Drug Use and Health: Detailed Tables . (2017). Accessed: July 4, 2017: http://www.samhsa.gov/data/sites/default/files/NSDUH-DetTabs-2016/NSDUH-DetTabs-2016.pdf.

13. Treatment Episode Data Set (TEDS) 2004 - 2014. National Admissions to Substance Abuse Treatment Services. (2016). Accessed: November 25, 2019: http://wwwdasis.samhsa.gov/dasis2/teds_pubs/2014_teds_rpt_natl.pdf.

14. Schermer CR, Wisner DH: Methamphetamine use in trauma patients: a population-based study . J Am Coll Surg. 1999, 189:442-49. 10.1016/s1072-7515(99)00188-x

15. Hsu DJ, McCarthy EP, Stevens JP, Mukamal KJ: Hospitalizations, costs and outcomes associated with heroin and prescription opioid overdoses in the United States 2001-12. Addiction. 2017, 112:1558-64. 10.1111/add.13795

16. Borders TF, Booth BM, Han X, Wright P, Leukefeld C, Falck RS, Carlson RG: Longitudinal changes in methamphetamine and cocaine use in untreated rural stimulant users: racial differences and the impact of methamphetamine legislation. Addiction. 2008, 103:800-808. 10.1111/j.1360-0443.2008.02159.x

17. McCabe SE, Morales M, Cranford JA, Delva J, McPherson MD, Boyd CJ: Race/ethnicity and gender differences in drug use and abuse among college students. J Ethn Subst Abuse. 2007, 6:75-95. 10.1300/J233v06n02_06

18. Schmidt L, Greenfield T, Mulia N: Unequal treatment: racial and ethnic disparities in alcoholism treatment services. Alcohol Res Health. 2006, 29:49-54.

19. Williams DR, Mohammed SA, Leavell J, Collins C: Race, socioeconomic status, and health: complexities, ongoing challenges, and research opportunities. Ann N Y Acad Sci. 2010, 1186:69-101. 10.1111/j.17496632.2009.05339.x

20. Druss BG, von Esenwein SA: Improving general medical care for persons with mental and addictive disorders: systematic review. Gen Hosp Psychiatry. 2006, 28:145-53. 10.1016/j.genhosppsych.2005.10.006

21. Schulte MT, Hser YI: Substance use and associated health conditions throughout the lifespan . Public Health Rev. 2014, 35:3. 10.1007/bf03391702

22. Hedegaard H, Miniño AM, Warner M: Drug overdose deaths in the United States, 1999-2017. NCHS Data Brief. 2018, 329:1-8.

23. Young NK, Gardner S, Otero C, Dennis K, Chang R, Earle K, Amatetti S: Substance-exposed Infants: State Responses to the Problem. HHS Pub. No. (SMA) 09-4369. Young NK, Gardner S, Otero C, Dennis K, Chang R, Earle K, Amatetti S (ed): Substance Abuse and Mental Health Services Administration, Rockville; 2009.

24. Creanga AA, Sabel JC, Ko JY, et al.: Maternal drug use and its effect on neonates: a population-based study in Washington State. Obstet Gynecol. 2012, 119:924-33. 10.1097/AOG.0b013e31824ea276

25. Whiteman VE, Salemi JL, Mogos MF, Cain MA, Aliyu MH, Salihu HM: Maternal opioid drug use during pregnancy and its impact on perinatal morbidity, mortality, and the costs of medical care in the United States. J Pregnancy. 2014, 2014:906723. 10.1155/2014/906723

26. Shei A, Rice JB, Kirson NY, et al.: Characteristics of high-cost patients diagnosed with opioid abuse . J Manag Care Spec Pharm. 2015, 21:902-12. 10.18553/jmcp.2015.21.10.902

27. Samet JH, Friedmann P, Saitz R: Benefits of linking primary medical care and substance abuse services: patient, provider, and societal perspectives. Arch Intern Med. 2001, 161:85-91. 10.1001/archinte.161.1.85

28. Quan H, Li B, Couris CM, et al.: Updating and validating the Charlson comorbidity index and score for risk adjustment in hospital discharge abstracts using data from 6 countries. Am J Epidemiol. 2011, 173:676-82. 10.1093/aje/kwq433

29. Heslin KC, Elixhauser A, Steiner CA: Hospitalizations involving mental and substance use disorders among adults, 2012: Statistical Brief 191 (Internet). Healthcare Cost and Utilization Project (HCUP) Statistical Briefs. Agency for Healthcare Research and Quality, Rockville; 2015. 1-14.

30. Brooner RK, King VL, Kidorf M, Schmidt CW Jr, Bigelow GE: Psychiatric and substance use comorbidity among treatment-seeking opioid abusers. Arch Gen Psychiatry. 1997, 54:71-80. 10.1001/archpsyc.1997.01830130077015 